\title{
Presynaptic feedback system of oxytocinergic neurons in the hypothalamus of a rat model of adjuvant arthritis
}

\section{Teruaki Fujitani}

University of Occupational and Environmental Health

\section{Takanori Matsuura}

University of Occupational and Environmental Health

Makoto Kawasaki ( $\square$ k-makoto@med.uoeh-u.ac.jp )

Sangyo Ika Daigaku Igakubu https://orcid.org/0000-0001-8410-5800

\section{Hitoshi Suzuki}

University of Occupational and Environmental Health

\section{Haruki Nishimura}

University of Occupational and Environmental Health

\section{Kazuhiko Baba}

University of Occupational and Environmental Health

\section{Yoshiaki Yamanaka}

University of Occupational and Environmental Health

\section{Hideo Ohnishi}

University of Occupational and Environmental Health

\section{Yoichi Ueta}

University of Occupational and Environmental Health

\section{Akinori Sakai}

University of Occupational and Environmental Health

\section{Research}

Keywords: chronic inflammation, feedback system, L-NAME, L-368,899, magnocellular, monomeric red fluorescent protein 1 , nitric oxide synthase, paraventricular nucleus, transgenic rat, whole cell patch clamp

Posted Date: December 10th, 2019

DOl: https://doi.org/10.21203/rs.2.18454/v1

License: (c) (1) This work is licensed under a Creative Commons Attribution 4.0 International License. Read Full License 


\section{Abstract}

The neurohypophysial hormone oxytocin (OXT) is synthesized in the hypothalamic paraventricular and supraoptic nuclei (PVN and SON, respectively). Recently, some studies have considered that OXT is important in sensory modulation, and that OXT protein is upregulated by acute and chronic nociception. However, the mechanism by which OXT is upregulated in neurons is unknown. In the present study, we examined the excitatory postsynaptic currents (EPSCs) in OXT-ergic neurons in the PVN in an adjuvant arthritis (AA) rat model, a model of chronic inflammation, using whole cell patch clamping. Transgenic rats expressed OXT and the monomeric red fluorescent protein 1 (mRFP1) fusion gene to visualize the OXT-ergic neurons, and the OXT-mRFP1 transgenic rat model of AA was developed by injection of the heat-killed Mycobacterium butyricum . Furthermore, the feedback system of synthesized OXT was also examined using the OXT receptor antagonist $(\mathrm{L}-368,899)$. We found that the frequency of miniature $(\mathrm{m})$ EPSCs and spontaneous (s) EPSCs in OXT-mRFP1 neurons in the PVN were significantly increased in AA rats. Furthermore, L-368,899 dose-dependently increased the frequency of mEPSCs and sEPSCs in OXTergic neurons. Following bath application of the GABA A receptor antagonist picrotoxin and cannabinoid receptor 1 antagonist AM 251, L-368,899 still increased the frequency of mEPSCs. However, following the bath application of the nitric oxide synthase (NOS) inhibitor (L-NAME), L-368,899 did not alter the mEPSC frequency. Thus, it is suggested that OXT-ergic neuron activity is upregulated via an increase in glutamate release, and the upregulated OXT neurons have a feedback system with released OXT. It is possible that NO but not GABA may contribute to the feedback system of OXT neurons in chronic inflammation.

\section{Introduction}

One of the neurohypophysial hormones, oxytocin (OXT), is synthesized in the hypothalamic paraventricular nucleus (PVN) and supraoptic nucleus (SON), and enters into the systemic circulation from the posterior pituitary. The functions of OXT are classically considered to be contraction of the uterus and the milk reflex during lactation. Recently, it has been shown that OXT is involved in maternal bonding, and sexual behavior and social affiliation (1)(2). Furthermore, some studies have suggested that OXT is important in sensory modulation (3). For example, OXT attenuates inflammation and myeloperoxidase activity induced by subcutaneous injection of carrageenan (4); subarachnoid administration of OXT lowers the threshold of pain (5); transient OXT administration abolishes hypersensitivity in the nerve injury rat model (6). In addition, it is known that the activity of OXT-ergic neurons is upregulated by various stresses, such as conditioned fear (environmental stimuli previously paired with foot shocks), unconditioned fear (intermittently applied foot shocks), noxious stimuli (7-9), and morphine withdrawal (10). A previous study reported that OXT was upregulated in the PVN, SON, posterior pituitary, and the dorsal horn of the spinal cord in rats with adjuvant arthritis (AA), which is a well-known chronic pain model (11). However, the mechanism by which activity is upregulated is unknown in OXT-ergic neurons. In vivo electrophysiological studies of rats and mice have demonstrated that peripheral chronic nociceptive stimulation and/or injury models produce evoked action potentials or 
excitatory presynaptic potentials (EPSPs) in the central nervous system (12); however, previous studies have not examined whether the mechanism involves OXT-ergic neurons.

The hypothalamic PVN generates a homeostatic response (13). It has been shown that neuroendocrineautonomic integration exists as a homeostatic response in the PVN (14), and the activity-dependent dendritic release from the PVN acts diffusely to increase the activity of pre-sympathetic neurons (13). The dendrites release OXT and OXT functions as a paracrine or autocrine signal at the site of its occurrence (15). However, the mechanism of communication between OXT-ergic neurons is little known.

In this study, we investigated EPSCs in OXT-ergic neurons in the PVN in an AA rat model using whole cell patch clamping. A previous study demonstrated that OXT mRNA could not be distinguished from vasopressin mRNA in magnocellular neuroendocrine cells in the hypothalamus using the polymerase chain reaction (PCR) method (16). Thus, in these experiments, we used transgenic rats that expressed OXT and the monomeric red fluorescent protein 1 (mRFP1) fusion gene to enable visualization of OXTergic neurons (17). The OXT-mRFP1 transgenic rat is useful to study the electrophysiology of the hypothalamo-neurohypophysial system because OXT neurons can be detected easily using fluorescence microscopy. We hypothesize that the synthesized OXT in AA rats affects the feedback system including EPSPs between OXT-ergic neurons. We investigated the detailed feedback system of synthesized OXT by using an OXT antagonist. In addition, we examined the contribution of retrograde synaptic transmitters in the feedback system of OXT-ergic neurons.

\section{Materials And Methods}

\section{Animals}

We used OXT-mRFP1 transgenic rats (Wistar, male, aged 6-8 weeks and weighing 200-300 g) that were bred and maintained as described previously (17). All rats received food and water ad libitum and were maintained on a 12:12 hour light / dark cycle (lights on 07.00 hours) at $22-25^{\circ} \mathrm{C}$ with three rats housed per plastic cage. PCR for genomic DNA was performed in all rats for confirmation of OXT-mRFP1-positive.

\section{Adjuvant Arthritis (AA) model}

We intracutaneously (i.c.) injected heat-killed Mycobacterium butyricum (1 mg/rat) in paraffin liquid to OXT-mRFP1 transgenic rats via their tail root (11). In the same manner, we injected $100 \mu \mathrm{l}$ of paraffin liquid i.c. via the tail root of control rats. The arthritis index (Al) was scored before decapitation. The severity of arthritis in paw of rats was graded from 0 to 4 . Grade 0 has no swelling. Grade 1 shows mild swelling or erythema on only one toe. Grade 2 indicates swelling of one or more toes. Grade 3 indicates swelling of the ankle. Grade 4 shows severe swelling of the arthritis of the toe and ankle. 


\section{Slice preparations}

The rats were killed by decapitation on day 15 post-injection (vehicle or heat-killed Mycobacterium butyricum in paraffin liquid). After removing each brain from the skull, it was confirmed that there was no significant hemorrhage or gross contusion. The brains were placed in cold oxygenated $\left(5 \% \mathrm{CO}_{2}\right.$ and $95 \%$ $\mathrm{O}_{2}$ ) cutting solution containing $252 \mathrm{mM}$ sucrose, $6 \mathrm{mM} \mathrm{MgSO}_{4}, 2.5 \mathrm{mM} \mathrm{KCl}, 25 \mathrm{mM}, 0.5 \mathrm{mM} \mathrm{CaCl}_{2}$, $1.2 \mathrm{mM} \mathrm{NaH}_{2} \mathrm{PO}_{4}, \mathrm{NaHCO}_{3}$, and $10 \mathrm{mM}$ glucose. We cut the brain including the hypothalamus and glued it with instant adhesive on the stage of a vibratome-type slicer (DSK Linearslicer PR07, Kyoto, Japan). As described previously, coronal slices (300 $\mu$ m thick) containing the PVN were cut after carefully removing the meninges (18). The slices were preincubated in artificial cerebrospinal fluid (ACSF) containing $124 \mathrm{mM} \mathrm{NaCl}, 2 \mathrm{mM} \mathrm{CaCl}_{2}, 2.5 \mathrm{mM} \mathrm{KCl}_{2} \mathrm{mM} \mathrm{MgSO}_{4}, 1 \mathrm{mM} \mathrm{NaH}_{2} \mathrm{PO}_{4}, 10 \mathrm{mM}$ glucose, and $25 \mathrm{mM}$ $\mathrm{NaHCO}_{3}$ at room temperature for at least $1 \mathrm{~h}$, and then the slices were transferred to the recording chamber (19).

\section{In vitro patch-clamp recordings}

We placed the brain slices onto a glass-bottomed chamber and fixed the brain slice with a grid of parallel nylon threads supported by the weight of C-shaped stainless-steel. A low-pressure aspiration system kept the solution level constant. To identify mRFP1 positive neurons, an upright microscope (BX-50, Olympus) and infrared differential interference contrast (DIC) optics with a mRFP1 filter (Olympus) were used. We acquired signals filtered at $3 \mathrm{kHz}$ with a patch-clamp amplifier (Axopatch 200B; Axon Instruments, Sunnyvale, CA, USA) and digitized at $1 \mathrm{kHz}$ with an analogue to digital converter (Digidata 1440A; Axon Instruments). Spontaneous EPSCs (sEPSCs) were recorded from OXT-mRFP1 neurons in the magnocellular $(\mathrm{m}) \mathrm{PVN}$ of the hypothalamus. The neurons were voltage-clamped at $-60 \mathrm{mV}$. We filled the recording pipettes (3-6 M $\Omega$ ) with a solution containing $145 \mathrm{mM} \mathrm{K}$-gluconate, $1 \mathrm{mM} \mathrm{MgCl}_{2}, 5 \mathrm{mM} \mathrm{NaCl}$, $10 \mathrm{mM}$ HEPES, $2 \mathrm{mM}$ Mg-ATP, $0.2 \mathrm{mM}$ EGTA, and $0.1 \mathrm{mM} \mathrm{Na}_{3}$-GTP (adjusted to $\mathrm{pH} 7.2$ with $\mathrm{KOH}$ ). The membrane potential was held at $-60 \mathrm{mV}$, except during these experiments. Using the property that glutamic voluntary quantum release is insensitive to $\mathrm{Na}+$ channel interception, we used tetrodotoxin (TTX) $(1 \mu \mathrm{mol})$ to separate miniature EPSCs (mEPSCs) from other currents.

\section{Drug application}

$\mathrm{GABA}_{\mathrm{A}}$ receptor antagonist (picrotoxin), OXT and Nw-Nitro-L-arginine methyl ester hydrochloride (LNAME) were obtained from Sigma Aldrich (St. Louis, USA). The OXT receptor antagonist (L-368,899) and cannabinoid receptor 1 antagonist (AM 251) were obtained from Tocris Cookson (Bristol, UK). Immediately before use, all using drugs were diluted from stock solutions to the final desired concentration in ACSF.

\section{Data analysis}


The data were collected and analyzed with Clampex 10.7 and Clampfit 10.7 (Molecular Devices, San Jose, CA, USA). An unpaired student's t-test, paired t-test, or one-way analysis of variance (ANOVA) were used for comparisons between two groups. The data are shown as means \pm standard error of the mean (SEM). In all cases, differences with $p<0.05$ were deemed to be statistically significant.

\section{Results}

\section{In vitro whole-cell patch-clamp recording of the hypothalamic mPVN in the OXT-mRFP1 transgenic rat}

Whole-cell patch-clamp recordings of OXT-mRFP1 neurons were performed in the mPVN of OXT-mRFP1 transgenic rats (Fig. 1A left). The mRFP1 fluorescence was observed in the PVN by fluorescence microscopy (Fig. 1A right). The OXT-mRFP1 neurons were distinguishable from other neurons by DIC fluorescence in the PVN (Fig. 1B a-f).

\section{Electrophysiological response of OXT-mRFP1 neurons in the PVN using whole-cell patch-clamp recordings}

We confirmed Al to evaluate the degree of inflammation on the day of the experiment. Al of AA rats was significantly higher than in control rats (control group: $0, n=6$ rats; AA group: $11.83 \pm 1.14, n=6$ rats; oneway ANOVA, $F_{1,10}=108.17, p=0.0000011$, Fig. $\left.2 A\right)$. We examined the resting membrane potential using whole-cell patch-clamp recordings of neurons in the PVN, and the average value of the resting membrane potential was $-63.10 \pm 3.29 \mathrm{mV}$ for controls and $-54.33 \pm 2.25 \mathrm{mV}$ for AA rats, and a significant difference was recognized (control group: $n=15$ neurons / 7 rats; AA group: $n=19$ neurons / 8 rats; oneway ANOVA, $F_{1,32}=5.13, p=0.030$, Fig. $2 B$ ). We show the action potential firing pattern of an OXT-mRFP1 neuron in current-clamp mode by induction of currents (from - $5 \mathrm{pA}$ to $+25 \mathrm{pA}$ with $5 \mathrm{pA}$ ) for $400 \mathrm{~ms}$ (Fig. 2C) and representative pooled results show neuronal responses to current induction (from - $10 \mathrm{pA}$ to $+40 \mathrm{pA}$ with $5 \mathrm{pA}$ ) in control and AA rats. There was no significant difference in the firing rate between the control and AA groups (each group: $n=12$ neurons / 6 rats). To examine whether excitatory presynaptic transmitter release changed in OXT-ergic neurons after chronic inflammation, we recorded sEPSCs and mEPSCs in OXT-mRFP1 neurons in the mPVN of control and AA rats. We show representative sEPSCs and mEPSCs recorded in the OXT-mRFP1 neurons in slices from control and AA rats at a holding potential of $-60 \mathrm{mV}$, and a cumulative histogram of inter-event interval and amplitude (Fig. 3A, B, D, E). The frequency of mEPSCs and sEPSCs in OXT-mRFP1 neurons in the mPVN significantly increased in the AA group compared with the control group (sEPSC control group: $1.28 \pm 0.16 \mathrm{~Hz}, \mathrm{n}=13$ neurons / 6 rats; sEPSC AA group: $3.02 \pm 0.48, n=12$ neurons $/ 6$ rats; one-way ANOVA, $F_{1,10}=12.59, p=$ 0.0017, Fig. $3 \mathrm{C}$ left) (mEPSC control group: $0.67 \pm 0.082 \mathrm{~Hz}, \mathrm{n}=34$ neurons / 9 rats; mEPSC AA group: $0.88 \pm 0.057, n=36$ neurons / 9 rats; one-way ANOVA, $F_{1,68}=4.53, p=0.037$, Fig. $3 F$ left). The amplitude of mEPSCs and sEPSCs in OXT-mRFP1 neurons in the mPVN were not significantly different between control and AA groups (Fig. $3 \mathrm{C}$ and $3 \mathrm{~F}$ right). 
Next, we investigated whether synthesized OXT from AA rats affects the EPSPs in OXT-ergic neurons. We used a bath application of the OXT receptor antagonist L-368,899 (20). The results show representative sEPSCs and mEPSCs recorded in OXT-mRFP1 neurons in slices from control and AA rats at a holding potential of $-60 \mathrm{mV}$ (Fig. 4A and D). Bath application of L-368,899 dose-dependently increased the frequency of mEPSCs and sEPSCs in OXT-mRFP1 neurons in AA rats (sEPSC; $10 \mathrm{nM}: 111.30 \pm 11.68 \%$ of baseline, $\mathrm{t}(10)=0.96, \mathrm{p}=0.38 ; 100 \mathrm{nM}: 145.58 \pm 24.11 \%$ of baseline, $\mathrm{t}(10)=1.89, \mathrm{p}=0.12 ; 1 \mu \mathrm{M}: 154.37$ $\pm 14.85 \%$ of baseline, $\mathrm{t}(10)=3.66, \mathrm{p}=0.014$, paired t-test, $\mathrm{n}=6$ neurons $/ 3$ rats, Fig. 4B right) (mEPSC; 10 $n M: 102.66 \pm 9.36 \%$ of baseline, $t(10)=0.28, p=0.79 ; 100 \mathrm{nM}: 169.05 \pm 16.09 \%$ of baseline, $t(10)=4.08$, $p=0.015 ; 1 \mu \mathrm{M}: 235.24 \pm 39.14 \%$ of baseline, $t(10)=3.46, p=0.026$, paired t-test, $n=6$ neurons $/ 3$ rats, Fig. 4E right); however, there was no change in control rats (sEPSC; $10 \mathrm{nM}: 107.99 \pm 9.87 \%$ of baseline, $t$ $(10)=0.81, p=0.45 ; 100 \mathrm{nM}: 108.23 \pm 8.53 \%$ of baseline, $t(10)=7.74, p=0.96 ; 1 \mu \mathrm{M}: 103.39 \pm 6.96 \%$ of baseline, $\mathrm{t}(10)=0.48, \mathrm{p}=0.64$, paired t-test, $\mathrm{n}=6$ neurons $/ 3$ rats, Fig. 4B left) (mEPSC; $10 \mathrm{nM}$ : $99.01 \pm$ $8.80 \%$ of baseline, $t(10)=0.11, p=0.91 ; 100 n M: 105.46 \pm 13.54 \%$ of baseline, $t(10)=0.40, p=0.69 ; 1$ $\mu \mathrm{M}: 101.91 \pm 8.77 \%$ of baseline, $\mathrm{t}(10)=0.22, \mathrm{p}=0.82$, paired t-test, $\mathrm{n}=6$ neurons $/ 3$ rats, Fig. 4E left). Bath application of L-368,899 did not change the amplitude of mEPSCs and sEPSCs in OXT-mRFP1 neurons in control and AA rats (Fig. $4 \mathrm{C}$ and F). Therefore, the results suggest that feedback of mEPSCs and sEPSCs from synthesized OXT occurs in OXT-ergic neurons in AA rats.

\section{Examination of retrograde transmitters in the feedback system in OXT-mRFP1 neurons in AA rats}

Previous studies have demonstrated that regarding feedback in central neurons, retrograde synaptic transmission via transmitters such as nitric oxide (NO), cannabinoid receptor $1\left(\mathrm{CB}_{1}\right)$, and GABA is known to occur $(21-23)$. Thus, we investigated whether these transmitters were involved in the feedback from synthesized OXT in AA rats. Following bath application of the $\mathrm{GABA}_{A}$ receptor antagonist $(100 \mu \mathrm{M}$ picrotoxin) (Fig. 5A and B) and $\mathrm{CB}_{1}$ antagonist ( $2 \mu \mathrm{M}$ AM 251) (Fig. $5 \mathrm{C}$ and D), bath application of L368,899 still increased the frequency of mEPSCs (picrotoxin; $100 \mu \mathrm{M}$ picrotoxin: $125.41 \pm 10.7 \%$ of baseline, $t(10)=2.52, p=0.040,10 n M ~ L-368,899: 129.25 \pm 7.20 \%$ of baseline, $t(10)=4.06, p=0.0066$;

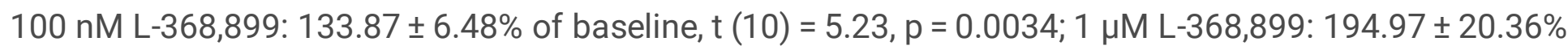
of baseline, $t(10)=4.66, p=0.00522$, paired t-test, $n=6$ neurons $/ 3$ rats, Fig. 4B left) (AM 251; $2 \mu M$ AM 251: $111.53 \pm 8.28 \%$ of baseline, $t(10)=1.39, p=0.23,10 \mathrm{nM} \mathrm{L}-368,899: 117.97 \pm 13.87 \%$ of baseline, $t$

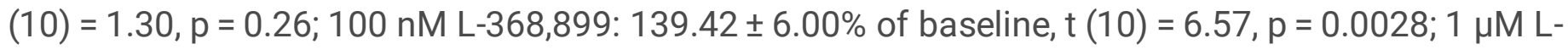
368,899: $215.11 \pm 20.70 \%$ of baseline, $t(10)=5.56, p=0.00512$, paired $t$-test, $n=6$ neurons $/ 3$ rats, Fig. 4D left), and did not change the amplitude of mEPSCs from baseline (Fig. 4B right and $\mathrm{D}$ right). However, following the bath application of the NOS inhibitor (100 $\mu \mathrm{M}$ L-NAME), bath application of L368,899 did not change the frequency (L-NAME; $100 \mu \mathrm{M}$ L-NAME: $99.94 \pm 4.39 \%$ of baseline, $\mathrm{t}(10)=0.08$, $p=0.94,10 \mathrm{nM} \mathrm{L-368,899:} 107.30 \pm 5.80 \%$ of baseline, $t(10)=1.26, p=0.26 ; 100 \mathrm{nM}$ L-368,899: $101.48 \pm$ $11.34 \%$ of baseline, $t(10)=0.13, p=0.90 ; 1 \mu \mathrm{M} \mathrm{L}-368,899: 106.34 \pm 8.15 \%$ of baseline, $t(10)=0.778, p=$ 0.48 , paired t-test, $n=6$ neurons / 3 rats, Fig. 4F left) or amplitude of mEPSCs in AA rats Fig. 4F right). 
Furthermore, mEPSCs increased significantly from baseline only following the bath application of picrotoxin (paired t-test, $p<0.05$ ). Therefore, this suggests that NOS contributes to the feedback system of synthesized OXT in AA rats.

\section{Confirmation of the effect of OXT and the OXT receptor antagonist L-368,899 in OXT-mRFP1neurons in slices from control rats}

We confirmed the effects of OXT and L-368,899 in OXT-mRFP1 neurons. After recording a stable baseline at a holding potential of $-60 \mathrm{mV}$, we recorded mEPSCs following the application of $1 \mu \mathrm{M}$ OXT and different doses of L368,899 (10 and $100 \mathrm{nM}$ and $1 \mu \mathrm{M}$ ) on mEPSCs in OXT-mRFP1 neurons in control rats. Following bath application of OXT, the frequency of mEPSCs in OXT-mRFP1 neurons was significantly increased (OXT; $1 \mu \mathrm{M}$ OXT: $233.45 \pm 25.99 \%$ of baseline, $t(10)=5.1, p=0.00215, n=6$ neurons / 4 rats, Fig. 6 left), while OXT did not change the amplitude of mEPSCs (Fig. 6 right). After application of L-368,899, the increased frequency of mEPSCs returned to baseline in a dose-dependent manner (10 nM L-368,899: $210.16 \pm 18.37 \%$ of baseline, $t(10)=5.99, p=0.00185 ; 100 \mathrm{nM} \mathrm{L-368,899:}$ $125.75 \pm 18.81 \%$ of baseline, $t(10)=1.37, p=0.22 ; 1 \mu \mathrm{M} \mathrm{L-368,899:} 98.66 \pm 10.17 \%$ of baseline, $t(10)=$ $0.13, p=0.90$, paired t-test, $n=6$ neurons $/ 3$ rats, Fig. 6 left), but the amplitude of mEPSCs remained unchanged (Fig. 6 right).

We confirmed that OXT affects the pre-synaptic current in OXT-mRFP1 neurons in control rats, and L368,899 inhibited the effect of OXT.

A hypothetical scheme for the excitatory system and feedback mechanism of OXT-ergic neurons in the hypothalamus of the chronic inflammation rat model is shown in Fig. 7.

\section{Discussion}

In the present study, we investigated mEPSCs and sEPSCs in OXT-ergic neurons in the mPVN by using OXT-mRFP1 transgenic rats with AA. We have demonstrated that the frequency of mEPSCs and sEPSCs was increased in OXT-mRFP1 neurons in the MPVN of AA rats. Furthermore, the feedback system of synthesized OXT was also investigated using L-368,899. L-368,899 dose-dependently increased the frequency of mEPSCs and sEPSCs further in the neurons of AA rats. Following the bath application of picrotoxin and AM 251, L-368,899 still increased the frequency of mEPSCs in AA rats. However, following the bath application of L-NAME, L-368,899 did not change the frequency of mEPSCs in AA rats. Our results indicate that the activity of OXT-ergic neurons is upregulated by increasing glutamate release in AA rats, and upregulated OXT neurons have a feedback system with released OXT. It is possible that NO but not GABA may contribute to the feedback system in OXT neurons in AA rats. 
A previous study demonstrated that OXT and mRFP1 mRNA and the red fluorescence intensity of mRFP1 were increased in the PVN of rats at 15 and 22 days after the onset of arthritis in OXT-mRFP1 transgenic rats. OXT-mRFP1 fluorescence intensity has also been shown to be increased in the dorsal horn of the spinal cord and posterior pituitary in AA rats (11). These results suggest that OXT was upregulated in both the hypothalamo-neurohypophysial and hypothalamo-spinal pathways by chronic inflammation, and these results are consistent with the increased frequency of mEPSCs and SEPSCs in this study. Therefore, OXT-mRFP1 neurons may be activated by pre-synaptic glutamate release.

We also found that L-368,899 dose-dependently increased the frequency of mEPSCs and sEPSCs in OXTmRFP1 neurons in AA rats, suggesting that the activity of presynaptic excitatory neurons was suppressed by increasing OXT in the AA rat. In this study, it is unclear which OXT-ergic neurons secrete OXT. It is possible that one of the major pathways involved in the secretion of OXT is paracrine (24), which is thought to enter the ventricle and act on the OXT receptor after release from the PVN (25). OXT is also synthesized in the parvocellular PVN neurons, and some of these neurons are thought to project to the magnocellular PVN OXT neurons (3). Consequently, it is assumed that the synthesized OXT suppressed the excitatory neurotransmission in the surrounding or its own OXT neurons in AA rats as a feedback system. In previous studies, it was unclear how feedback was provided to OXT neurons in the central nervous system. At the spinal level, OXT activates the pre-synaptic OXT receptor at the end of glutamatergic interneurons and promotes synaptic release of glutamate and inhibitory GABA-ergic interneurons (26). Therefore, OXT may activate excitatory and inhibitory neurons by stimulating the presynaptic OXT receptor in the central nervous system.

Meanwhile, there is a mechanism using a retrograde transmitter as a mechanism for feedback through the post-synaptic OXT receptor. NO has been shown to be present in the central nervous system (27) and has long been considered a retrograde neurotransmitter because it is a fat-soluble gas that diffuses across cell membranes (28). Neuronal NOS (nNOS) is abundant in the PVN, and nNOS in the PVN has been shown to be increased several rat models of stress (29)(30)(31). It has been suggested that NO plays a role as an important messenger in the PVN-spinal pathway and may act in concert with OXT (32).

NO synthesis is competitively inhibited by L-NAME, which has a similar structure to L-arginine (33). LNAME suppresses hippocampal long-term potentiation, and it has been reported that NO may be a retrograde transmitter of the hippocampus (34). On the other hand, it has been suggested that NO is also associated with signaling via the OXT receptor. Exogenous OXT hyperpolarizes dorsal root ganglia neurons that induce the feeling of pain in rats, and this hyperpolarization is mediated by the $\mathrm{Ca}^{2+} / \mathrm{nNOS}^{2}$ / NO / $\mathrm{K}_{\text {ATP }}$ pathway (35). NO is also associated with other neuronal signal pathways, such as the NO / sGC / cGMP pathway, and down-regulation of NMDA-receptor activity by reaction with thiol group(s) of the NMDA receptor's redox modulatory site (36) (37). Thus, released OXT may cause hyperpolarization and down-regulation of NMDA-receptor activity in several putative pathways in AA rats. As a result, OXT may modulate glutamine release though the OXT receptor in OXT-mRFP1 neurons. Pre-treatment with the OXT receptor antagonist Atociban or the selective NOS inhibitor N-propyl-L-arginine significantly attenuates the hyperpolarization caused by OXT (35). 
We found that OXT-ergic neurons generate negative feedback with OXT itself in the PVN in AA rats. In addition, $\mathrm{NO}$ is a contributor in the feedback-mechanism.

Although we investigated the contribution of $\mathrm{GABA}_{A}$ to the feedback system though OXT receptors, increased mEPSCs induced by the OXT receptor antagonist did not change following the bath application of the $G_{A B A}$ antagonist. It is thought that $G_{A B A}$ is not related with the feedback system through OXT receptors. Instead, $\mathrm{GABA}_{A}$ may work as a retrograde transmitter in other feedback systems that are not related to the OXT receptor.

A limitation of the study is that the evoked EPSC was not investigated. Thus, we did not examine postsynaptic changes of OXT-ergic neurons in AA rats. Moreover, the relationship between OXT-ergic neurons was not described. In the future, the plasticity of OXT-mRFP1 neurons should be examined, and paired recordings with OXT-mRFP1 neurons should be conducted.

In conclusion, the activity of OXT-ergic neurons is upregulated by increasing glutamate release in AA rats, and OXT neurons have a feedback system with released OXT. It is possible that NOS may contribute to the feedback system of in OXT-ergic neurons.

\section{List Of Abbreviations}

Oxytocin, OXT; paraventricular, PVN; supraoptic nuclei, SON; adjuvant arthritis, AA; monomeric red fluorescent protein 1, mRFP1; polymerase chain reaction, PCR; arthritis index, Al; artificial cerebrospinal fluid, ACSF; magnocellular, m; tetrodotoxin, TTX; differential interference contrast, DIC; N $\omega$-Nitro-L-arginine methyl ester hydrochloride, L-NAME; nitric oxide, NO; cannabinoid receptor $1, \mathrm{CB}_{1}$; nitric oxide synthase, NOS.

\section{Declarations}

\section{Ethics approval and consent to participate}

All procedures described in this study were performed under the control of the Animal Care and Laboratory Ethics Committee University of Occupational and Environmental Health, Japan, and followed the guidelines for laboratory animal use and management established by the Physiological Society of Japan.

\section{Consent for publication}

Not applicable.

\section{Availability of data and materials}

Data sets currently under investigation and / or analysis are available from corresponding authors on reasonable request. 


\section{Competing interests}

The authors declare that they have no competing interests.

\section{Funding}

This work was supported by grants from JSPS KAKENHI Grant Number JP $18 \mathrm{H} 06268$ to TM and (C) $19 K 09564$ to MK.

\section{Authors' contributions}

TM designed the project. TF and TM performed the electrophysiological experiments. TF, TM, and MK wrote the initial draft and finished the final version of the manuscript. HS, HN, KB, YY, HS, YU, and AS contributed to the analysis and interpretation of data and assisted in the preparation of the manuscript. All authors read and approved the final manuscript.

\section{Acknowledgments}

We thank Ms. Yuki Nonaka (University of Occupational and Environmental Health, Kitakyushu, Japan) for her technical assistance.

\section{References}

1. Higashida H, Lopatina O, Yoshihara T, Pichugina YA, Soumarokov AA, Munesue T, et al. Oxytocin signal and social behaviour: Comparison among adult and infant oxytocin, oxytocin receptor and CD38 gene knockout mice. J Neuroendocrinol. 2010;22:373-9.

2. Pedersen CA, Vadlamudi SV, Boccia ML, Amico JA. Maternal behavior deficits in nulliparous oxytocin knockout mice. Genes, Brain Behav. 2006;5:274-81.

3. Eliava M, Melchior M, Knobloch-Bollmann HS, Wahis J, da Silva Gouveia M, Tang Y, et al. A new population of parvocellular oxytocin neurons controlling magnocellular neuron activity and inflammatory pain processing. Neuron. 2016;89:1291-304.

4. Petersson $\mathrm{M}$, Wiberg $\mathrm{U}$, Lundeberg $\mathrm{T}$, Uvnäs-Moberg $\mathrm{K}$. Oxytocin decreases carrageenan induced inflammation in rats. Peptides. 2001;22:1479-84.

5. DeLaTorre S, Rojas-Piloni G, Martínez-Lorenzana G, Rodríguez-Jiménez J, Villanueva L, Condés-Lara M. Paraventricular oxytocinergic hypothalamic prevention or interruption of long-term potentiation in dorsal horn nociceptive neurons: Electrophysiological and behavioral evidence. Pain. 2009;144:3208.

6. Houle T, Ph D, Eisenach JC, Forest W, Salem W, Salem W, et al. Reversal of peripheral nerve injuryinduced hypersensitivity in the postpartum period: role of spinal oxytocin. Anesthesiology. 2014;118:1-16. 
7. Onaka T, Palmer JR, Yagi K. A selective role of brainstem noradrenergic neurons in oxytocin release from the neurohypophysis following noxious stimuli in the rat. Neurosci Res [Internet]. 1996;25:6775. Available from: https://linkinghub.elsevier.com/retrieve/pii/0168010296010292

8. Onaka T. Catecholaminergic mechanisms underlying neurohypophysial hormone responses to unconditioned or conditioned aversive stimuli in rats. Exp Physiol [Internet]. 2000;85:101s-110s. Available from: http://doi.wiley.com/10.1111/j.1469-445X.2000.tb00013.x

9. Onaka T. Neural pathways controlling central and peripheral oxytocin release during stress. $J$ Neuroendocrinol. 2004;16:308-12.

10. Russell J, Leng G, Bicknell R. Opioid tolerance and dependence in the magnocellular oxytocin system: a physiological mechanism? Exp Physiol. 1995;80:307-40.

11. Matsuura T, Kawasaki M, Hashimoto H, Ishikura T, Yoshimura M, Ohkubo J-I, et al. Fluorescent visualisation of oxytocin in the hypothalamo-neurohypophysial/-spinal pathways after chronic inflammation in oxytocin-monomeric red fluorescent protein 1 transgenic rats. J Neuroendocrinol [Internet]. 2015 [cited 2019 Jan 16];27:636-46. Available from:

http://doi.wiley.com/10.1111/jne.12290

12. Koga K, Shimoyama S, Yamada A, Furukawa T, Nikaido Y, Furue H, et al. Chronic inflammatory pain induced GABAergic synaptic plasticity in the adult mouse anterior cingulate cortex. Mol Pain. 2018;14.

13. Son SJ, Filosa JA, Potapenko ES, Biancardi VC, Zheng H, Patel KP, et al. Dendritic peptide release mediates interpopulation crosstalk between neurosecretory and preautonomic networks. Neuron [Internet]. Elsevier Inc.; 2013;78:1036-49. Available from: http://dx.doi.org/10.1016/j.neuron.2013.04.025

14. Stern JE. Neuroendocrine-autonomic integration in the paraventricular nucleus: Novel roles for dendritically released neuropeptides. J Neuroendocrinol. 2015;27:487-97.

15. Bealer SL, Armstrong WE, Crowley WR. Oxytocin release in magnocellular nuclei: Neurochemical mediators and functional significance during gestation. Am J Physiol - Regul Integr Comp Physiol. 2010;299.

16. Yamashita M, Glasgow E, Zhang BJ, Kusano K, Gainer H. Identification of cell-specific messenger ribonucleic acids in oxytocinergic and vasopressinergic magnocellular neurons in rat supraoptic nucleus by single-cell differential hybridization. Endocrinology. 2002;143:4464-76.

17. Katoh A, Fujihara H, Ohbuchi T, Onaka T, Hashimoto $T$, Kawata $M$, et al. Highly visible expression of an oxytocin-monomeric red fluorescent protein 1 fusion gene in the hypothalamus and posterior pituitary of transgenic rats. Endocrinology. 2011;152:2768-74.

18. Nagatomo T, Inenaga $\mathrm{K}$, Yamashita $\mathrm{H}$. Transient outward current in adult rat supraoptic neurones with slice patch-clamp technique: inhibition by angiotensin II. J Physiol. 1995;485:87-96.

19. Matsuura T, Li XH, Tao C, Zhuo M. Effects of matrix metalloproteinase inhibitors on N-methyl-Daspartate receptor and contribute to long-term potentiation in the anterior cingulate cortex of adult mice. Mol Pain. 2019;15:1-10. 
20. Evans BE, Leighton JL, Rittle KE, Gilbert KF, Lundell GF, Gould NP, et al. Orally active, nonpeptide oxytocin antagonists. J Med Chem. 1992;35:3919-27.

21. Di S, Maxson MM, Franco A, Tasker JG. Glucocorticoids regulate glutamate and GABA synapsespecific retrograde transmission via divergent nongenomic signaling pathways. J Neurosci. 2009;29:393-401.

22. Ohno-Shosaku T, Maejima T, Kano M. Endogenous cannabinoids mediate retrograde signals from depolarized postsynaptic neurons to presynaptic terminals. Neuron. 2001;29:729-38.

23. Zilberter $Y$, Harkany $T$, Holmgren CD. Dendritic release of retrograde messengers controls synaptic transmission in local neocortical networks. Neuroscientist. 2005;11:334-44.

24. Ludwig M, Pittman QJ. Talking back: Dendritic neurotransmitter release. Trends Neurosci. 2003;26:255-61.

25. Striepens N, Kendrick KM, Maier W, Hurlemann R. Prosocial effects of oxytocin and clinical evidence for its therapeutic potential. Front Neuroendocrinol [Internet]. Elsevier Inc.; 2011;32:426-50. Available from: http://dx.doi.org/10.1016/j.yfrne.2011.07.001

26. Breton JD, Veinante P, Uhl-Bronner S, Vergnano AM, Freund-Mercier MJ, Schlichter R, et al. Oxytocininduced antinociception in the spinal cord is mediated by a subpopulation of glutamatergic neurons in lamina I-II which amplify GABAergic inhibition. Mol Pain. 2008;4:1-12.

27. Garthwaite J, Charles SL, Chess-Williams R. Endothelium-derived relaxing factor release on activation of NMDA receptors suggests role as intercellular messenger in the brain. Nature [Internet]. 1988;336:385-8. Available from: http://www.nature.com/articles/336385a0

28. Kerwin JF, Lancaster JR, Feldman PL. Nitric oxide: A new paradigm for second messengers. J Med Chem. 1995;38:4343-62.

29. Bredt DS, Hwang PM, Snyder SH. Localization of nitric oxide synthase indicating a neural role for nitric oxide. Nature. 1990;347:768-70.

30. Calzà L, Giardino L, Ceccatelli S. NOS mRNA in the paraventricular nucleus of young and old rats after immobilization stress. Neuroreport [Internet]. 1993;4:627-30. Available from: https://insights.ovid.com/crossref?an=00001756-199306000-00006

31. Sánchez F, Moreno MN, Vacas P, Carretero J, Vázquez R. Swim stress enhances the NADPHdiaphorase histochemical staining in the paraventricular nucleus of the hypothalamus. Brain Res. 1999;828:159-62.

32. Nylén A, Skagerberg G, Alm P, Larsson B, Holmqvist B, Andersson KE. Nitric oxide synthase in the hypothalamic paraventricular nucleus of the female rat; organization of spinal projections and coexistence with oxytocin or vasopressin. Brain Res. 2001;908:10-24.

33. Rees DD, Palmer RMJ, Schulz R, Hodson HF, Moncada S. Characterization of three inhibitors of endothelial nitric oxide synthase in vitro and in vivo. Br J Pharmacol. 1990;101:746-52.

34. Schuman EM, Madison D V. A requirement for the intercellular messenger nitric oxide in long-term potentiation. Science [Internet]. 1991;254:1503-6. Available from:

http://www.ncbi.nlm.nih.gov/pubmed/1720572 
35. Gong L, Gao F, Li J, Li J, Yu X, Ma X, et al. Oxytocin-induced membrane hyperpolarization in painsensitive dorsal root ganglia neurons mediated by $\mathrm{Ca} 2+/ \mathrm{nNOS} / \mathrm{NO} / \mathrm{K}$ ATP pathway. Neuroscience. 2015;289:417-28.

36. Klein C. Nitric oxide and the other cyclic nucleotide. Cell Signal. 2002;14:493-8.

37. Lipton SA, Choi YB, Pan ZH, Lei SZ, Chen HSV, Sucher NJ, et al. A redox-based mechanism for the neuroprotective and neurodestructive effects of nitric oxide and related nitroso-compounds. Nature. 1993;364:626-32.

\section{Figures}


A

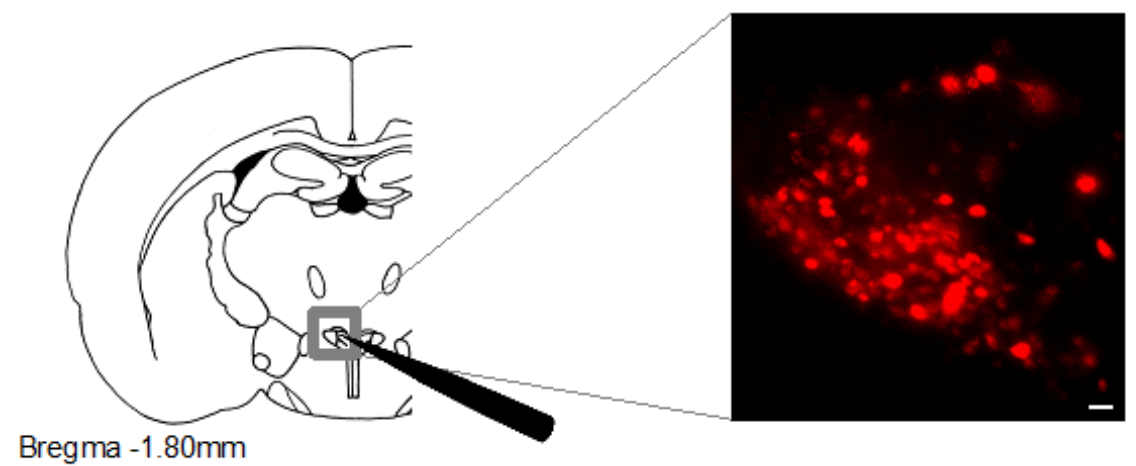

B
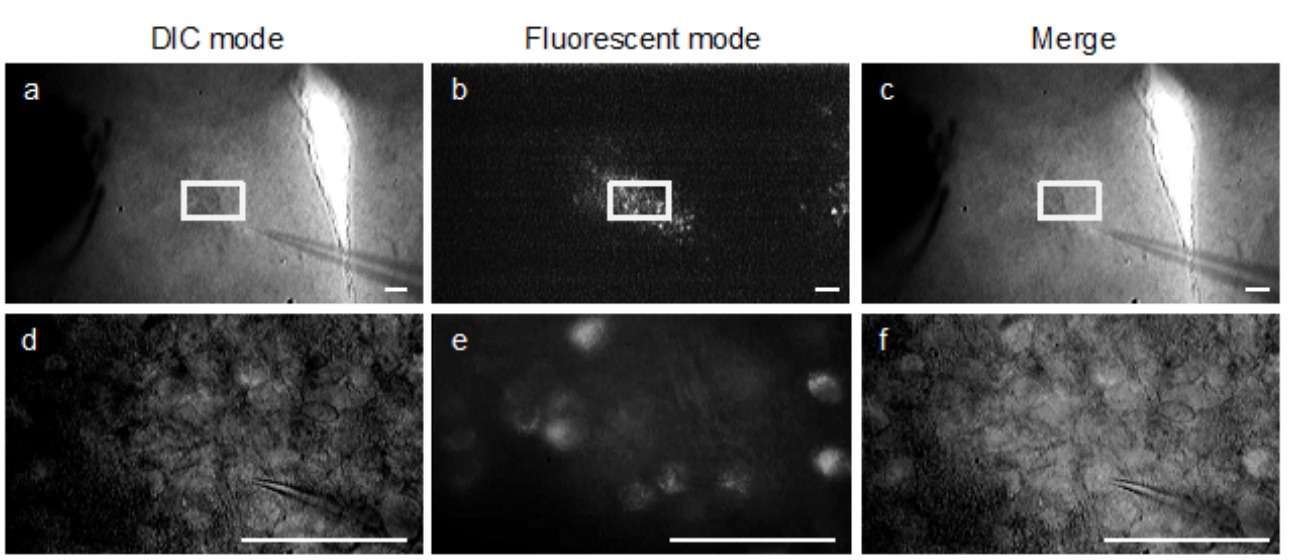

Fig.1

\section{Figure 1}

In vitro whole-cell patch-clamp recording of neurons in the hypothalamic PVN in the OXT-mRFP1 transgenic rat (A) Left: Schematic drawing of a coronal brain slice including the PVN. Right: OXT-mRFP1 neurons are shown in $300 \mu \mathrm{m}$ slice including the PVN using fluorescence microscopy. (B) OXT-mRFP1 neurons are distinguished from other neurons by differential interference contrast (DIC) and fluorescence 
microscopy. DIC mode (B-a, d), fluorescent mode (B-b, e), and the merged images (B-c, $f$ ) are shown. B-d-f are shown with high-magnified images of $B-a-c$ boxed regions. Scale bars $=100 \mu \mathrm{m}$ in A right and $B$.

\section{Figure 2}

Electrophysiological response of OXT-mRFP1 neurons in the magnocellular PVN using whole-cell patchclamp recordings $(\mathrm{A})$ The arthritis index (Al) in control and AA rats (control group: $n=6$ rats; AA group: $n=$ 6 rats. (B) Representative pooled results showing resting membrane potential $(\mathrm{mV})$ in control and AA rats (control group: $\mathrm{n}=15$ neurons / 7 rats; AA group: $\mathrm{n}=19$ neurons $/ 8$ rats). (C) Example AP firing pattern of an OXT-mRFP1 neuron in current-clamp mode by induction of currents (from -5 pA to +25 pA with 5 pA) for $400 \mathrm{~ms}$. (D) Representative pooled results showing neuronal responses to current injections (from $-10 \mathrm{pA}$ to $+40 \mathrm{pA}$ with $5 \mathrm{pA}$ ) in control and AA rats (each group, $\mathrm{n}=12$ neurons / 6 rats). $\# p<0.05$, \#\#p < 0.01 .

\section{Figure 3}

sEPSCs and mEPSCs recorded in the OXT-mRFP1 neurons in control and AA rats (A, D) Representative sEPSCs and mEPSCs recorded in the OXT-mRFP1 neuron in slices from a control rat (left) and AA rat (right) at a holding potential of $-60 \mathrm{mV}$. (B, E) Cumulative interevent interval (left) and amplitude (right) histograms of sEPSCs (B) and mEPSCs (E) recorded in slices from control rats (sEPSCs; $n=13$ neurons / 6 rats, $\mathrm{mEPSCs} ; \mathrm{n}=34$ neurons $/ 9$ rats) and AA rats (sEPSCs; $\mathrm{n}=12$ neurons $/ 6$ rats, $\mathrm{mEPSCs} ; \mathrm{n}=36$ neurons / 9 rats). (C, F) Summary frequency (left) and amplitude (right) of sEPSC and mEPSCs data. \#p < $0.05, \# \# p<0.01$. 

A sEPSCs

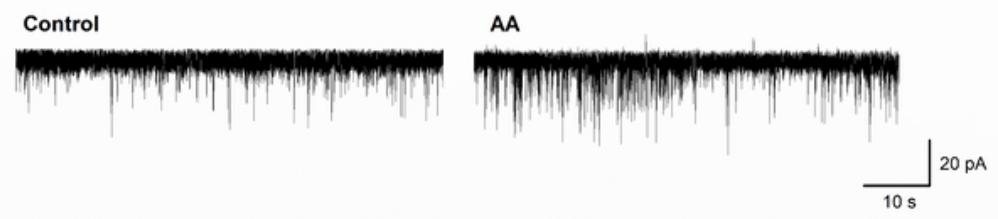

B

C
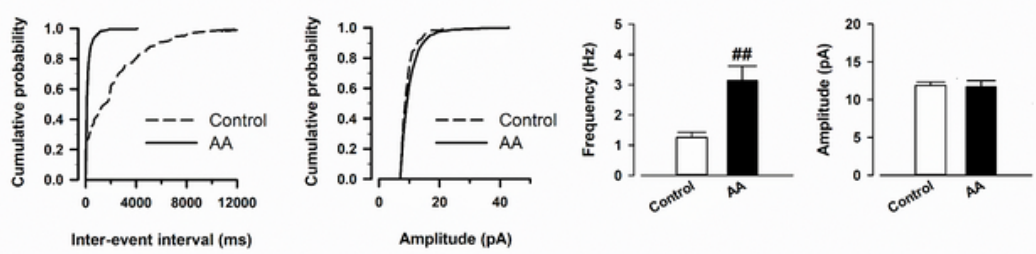

D mEPSCs
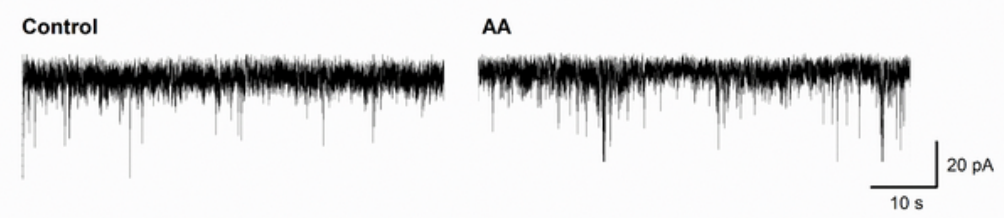

E
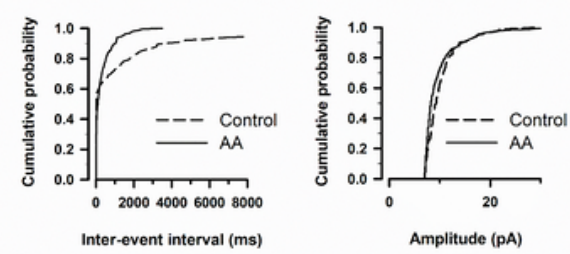

F
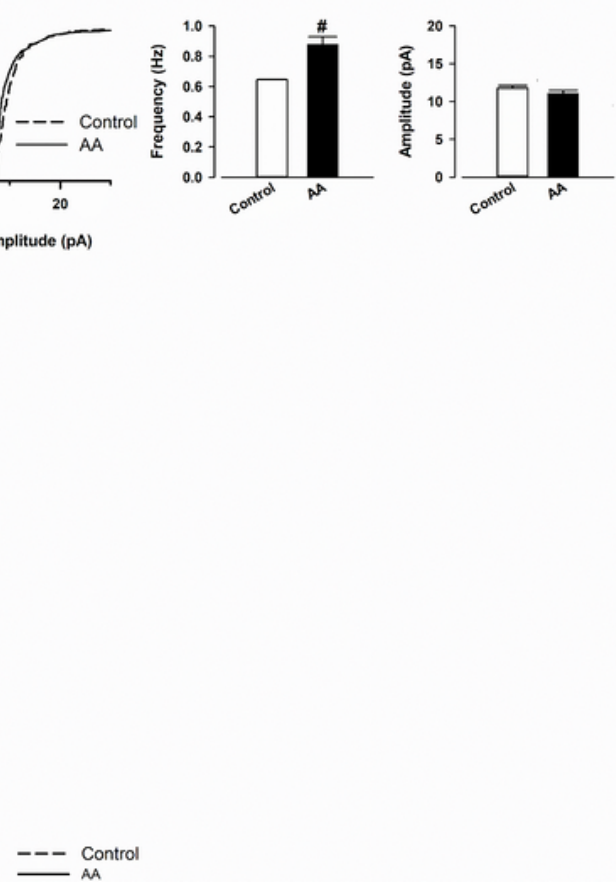

Figure 4

Effect of bath application of L368,899 on sEPSCs and mEPSCs in OXT-mRFP1 neurons in slices from control (left) and AA (right) rats (A, D) Example sEPSC (A) and mEPSC (D) traces are shown. (B, E) Summary frequency data of the different doses of L368,899 (10 and100 nM and $1 \mu \mathrm{M}$ ) on sEPSCs (B) and mEPSCs (E). (C, F) Summary amplitude data of the different doses of L368,899 (10 and $100 \mathrm{nM}$ and 
$1 \mu \mathrm{M}$ ) on sEPSCs (C) and mEPSCs ( $F)$. (each group, $n=6$ neurons $/ 3$ rats) $\# p<0.05$ compared with baseline, \#\#p < 0.01 compared with baseline.

\section{Figure 5}

Effect of bath application of picrotoxin, AM 251, and L-NAME on induced mEPSCs increased by L368,899 in OXT-mRFP1 neurons in slices from AA rats (A, C, E) Example trace of mEPSCs following the bath application of $100 \mu \mathrm{M}$ picrotoxin (A), $2 \mu \mathrm{M}$ AM 251 (C), and $100 \mu \mathrm{M}$ L-NAME (E) after the bath application of L-368,899 in OXT-mRFP1 neurons in AA rats are shown. (B, D, F) Summary frequency (left) and amplitude (right) data of the effect of picrotoxin (B), AM 251 (D), and L-NAME (F) after the bath application of L-368,899 in OXT-mRFP1 neurons in AA rats (each group, $n=6$ neurons / 3 rats). $\# p<0.05$, $\# \# p<0.01 .{ }^{*} p<0.05$ compared with only $100 \mu \mathrm{M}$ picrotoxin or $2 \mu \mathrm{M}$ AM 251.

\section{Figure 6}

Effect of bath application of OXT and L-368,899 on mEPSCs in OXT-mRFP1 neurons in slices from control rats $(A, C, E)$ Example trace of mEPSCs following the application of $1 \mu \mathrm{M}$ OXT and different doses of L368,899 (10 and $100 \mathrm{nM}$ and $1 \mu \mathrm{M}$ ) on mEPSCs in OXT-mRFP1 neurons in AA rats are shown. (B, D, F) Summary frequency (left) and amplitude (right) data of $1 \mu \mathrm{M}$ OXT and different doses of L368,899 (10 and $100 \mathrm{nM}$ and $1 \mu \mathrm{M}$ ) on mEPSCs in OXT-mRFP1 neurons in control rats. (each drug group, $\mathrm{n}=6$ neurons / 4 rats) $\# \# p<0.01$ 


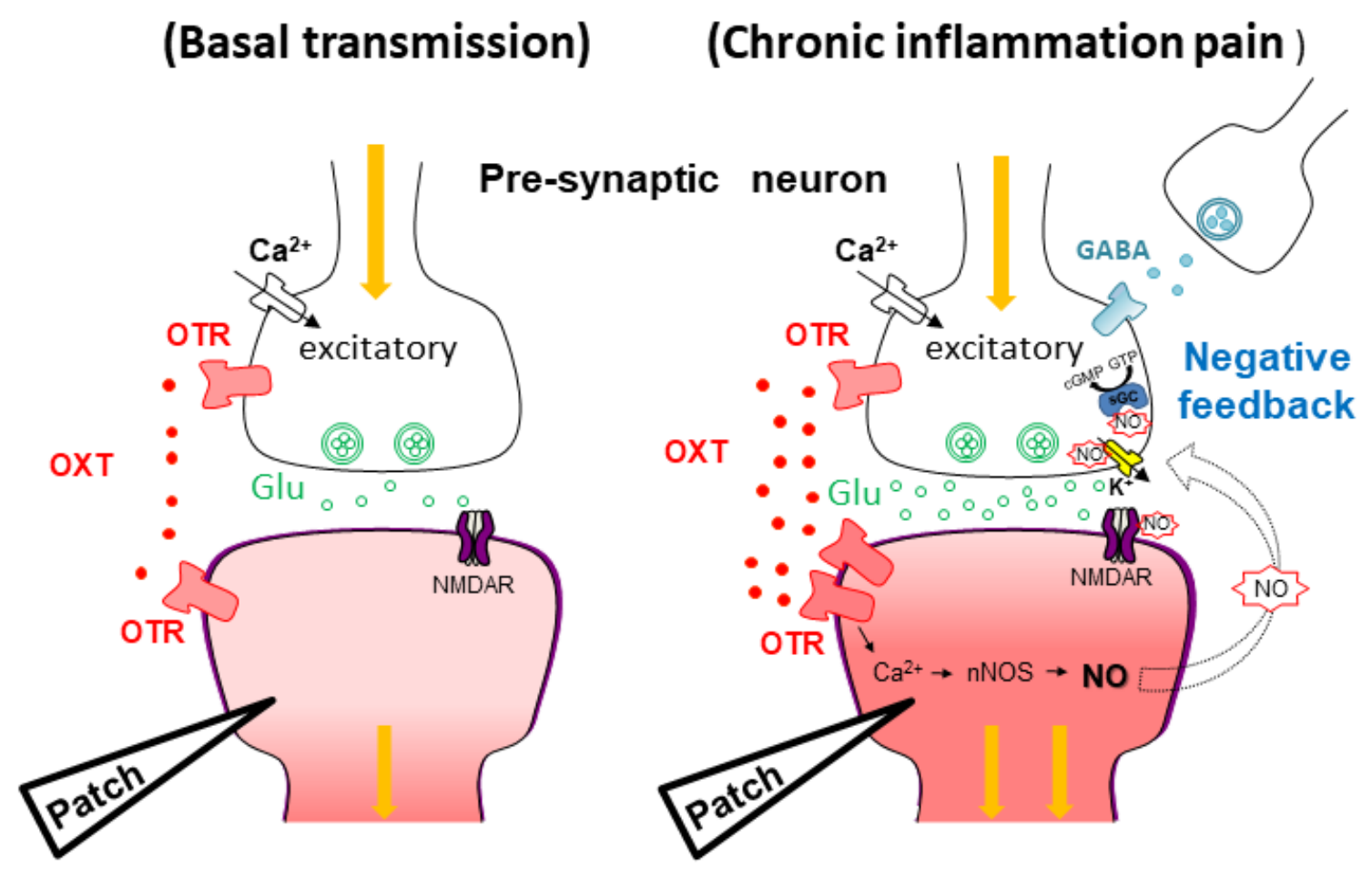

Post-synaptic OXT-ergic Neuron

Figure 7

A hypothetical scheme for the excitatory system and feedback mechanism of OXT-ergic neurons in the hypothalamus in the rat chronic inflammation model (Left) Basal transmission: Oxytocin (OXT) receptor (OTR) is present in both excitatory pre- and post-synaptic neurons in OXT-ergic neurons. (Right) Chronic inflammation pain: Chronic inflammation pain enhances excitatory synaptic transmission. Activated OXT works as negative feedback around itself via the OTR. The signaling pathway of the negative feedback 
system contributes neuronal nitric oxide synthase (nNOS) to promote nitric oxide (NO) production. NO and GABA act as retrograde neurotransmitters causing negative feedback at the pre-or post-synaptic OXTergic neurons. 